\title{
Real-Time Data Processing and Feedback During In-Situ Heating
}

\author{
Benjamin K. Miller ${ }^{1}$, Cory Czarnik ${ }^{1}$, George Vacek ${ }^{2}$, Stephen Mick ${ }^{1}$ \\ 1. Gatan Inc., Pleasanton, USA. \\ 2. DataDirect Networks, Chatsworth, USA.
}

Real-time processing of digitally acquired data dates back to at least the 1980s [1], but decades later, most data processing still takes place long after acquisition, on a separate computer. Implementing realtime processing is especially important for in-situ experiments, in which the next experimental step at the microscope is often determined by dynamic changes observed in the sample. Though a skilled operator can be incredibly good at picking up subtle changes in complex images, Gatan's latest in-situ cameras store more frames to disk than can be displayed even with high-end video cards. When using the fastest data collection rates, it is not possible for the experimentalist to monitor every acquired image. Although simple processing, like live FFTs can be used for analyzing data in real-time, only the subset of the acquired frames that are displayed on the computer screen are actually analyzed at the microscope.

This advent of fast cameras for capturing dynamic changes during in-situ experiments, requires a new approach to analyze the data in real-time. Gatan is working toward flexible solutions for live processing on the full stream of data from high-speed cameras. In this work, summarized in Figure 1, a scalable parallel file system with hundreds of terabytes of storage from DataDirect Networks (DDN) was used to save all data from a Gatan OneView IS camera as it was acquired during an in-situ heating experiment. While data was still being written to the filesystem, a 2nd computer running Gatan's GMS (Digital Micrograph) software was used to process the incoming data via scripting. In this case, the script converted a selected area electron diffraction pattern into a 1D profile, so that subtle changes could be more easily discerned by the TEM operator as shown in Figure 2. This processed data was again saved in DM4 format on the DDN file system and the analyzed data was accessed by GMS on the computer at the TEM. This analyzed data was continually updated and displayed next to the live view window. For the initial proof-of concept experiment, this processing loop took about 1 second for each $4 \mathrm{k} \times 4 \mathrm{k}$ diffraction pattern that was analyzed. Data was captured by the camera at 25 frames per second, so only 1 of every 25 image frames was processed, but this processing provided enough insight into the sample structure to enable the user to modify the experimental conditions in near-real-time based on the displayed results from the analysis.

This flexible and simple method for live data processing has great potential. The proof-of-concept experiment demonstrates a method in which the computational demands of the data analysis do not conflict with the demands of the data capture, since the two are performed on separate systems. This is enabled by the storage solution, which has the bandwidth to ingest all the incoming data from the camera, while still handling all the data traffic required for the live processing. The achieved near realtime feedback to the TEM operator enabled phase changes to be easily recognized during in-situ heating. The proof-of-concept experiment already performed could be extended by modifying the processing script so that any type of processing or analysis, including machine learning, model-based algorithms, and complex reconstructions could be performed. Since the data analysis was performed on a computer which does not interface with any experimental hardware, it is relatively simple to make changes to this part of the system. For example, the data analysis could instead be performed on a high performance 
computing platform, rather than a single desktop computer, speeding the analysis up to video frame rates and, if desired, to the full frame rate of the camera.

References:

[1] W. Krakow, Ultramicroscopy 18 (1985) p. 197.
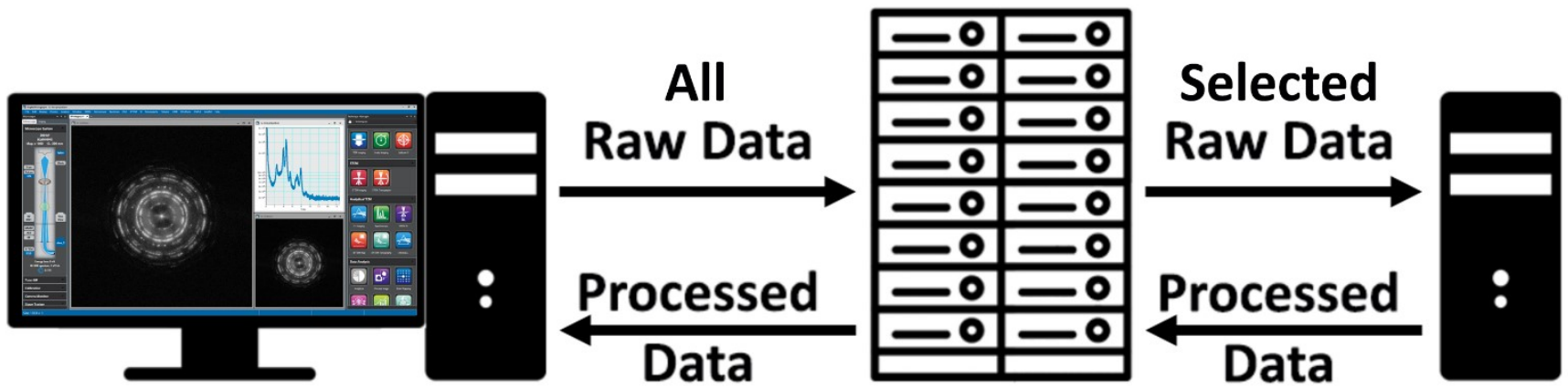

Figure 1. Schematic showing data flow. Raw data from the camera is stored to disk on the DDN storage system, from which it is read by a $2^{\text {nd }}$ computer. This processes the data, storing the processed data back to the DDN system, from which the TEM computer loads and displays the processed data.
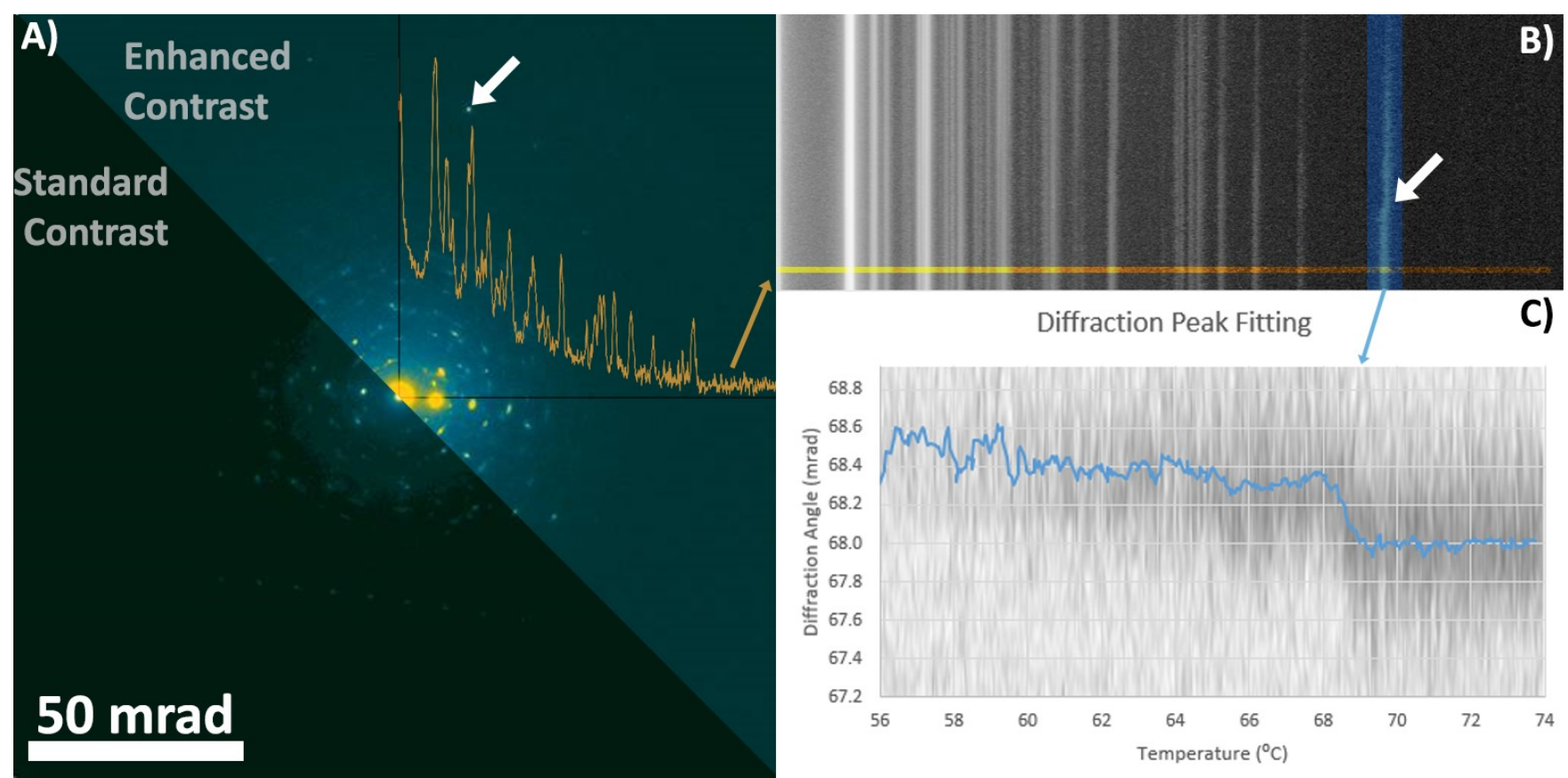

Figure 2. Results from a $\mathrm{VO}_{2}$ Monoclinic to Rutile phase transformation, demonstrating one application of this live processing. In this case, a subtle change in the diffraction pattern which is unlikely to be detected by the user in the raw 2D pattern (A) can be seen clearly in the processed data (B). The white arrow in A points out the diffraction spot that shifts slightly at the temperature marked by the arrow in B. The line plot in A shows a single processed pattern. Further, offline processing can be applied to fit the position of the selected diffraction peak as a function of time (and thus temperature), and this fit is shown in $\mathrm{C}$, with the raw data displayed in the background of the plot. 